\title{
Real-time monitoring of microfluidic lithography
}

\author{
Dario Pisignano *, Francesca Di Benedetto, Luana Persano, Elisa Mele, Roberto Cingolani \\ ${ }^{a}$ NNL, National Nanotechnology Laboratory of Istituto Nazionale di Fisica della Materia (INFM), clo Dipartimento di Ingegneria \\ dell 'Innovazione, Università di Lecce, via Arnesano, I-73100 Lecce, Italy
}

\begin{abstract}
In order to observe in real-time and to accurately measure the filling speed during the capillary process in planar microfluidic devices, we integrated a ruler in the silicon master structures, side by side with the channels, and replicated it into the elastomeric elements. The time dependence of the covered length $(z, t)$ and of the filling rate $(v, t)$ under different temperature and surface functionalization conditions was then exactly determined by optical microscopy. This allowed us to quantitatively assess the strong dependence of $v$ on the process environmental conditions, and to discard in real-time the presence of impurities on employed substrates, thus allowing one to strongly improve the throughput of the microfluidic-based soft lithography for nanotechnology applications.
\end{abstract}

Keywords: Microfluidics, polyurethane, polymer-polymer interfaces, interface preparation, control of interface structure and morphology.

\section{Introduction}

The need to pursue the miniaturisation trend predicted by the Moore's law is strongly acting as driving force for developing and implementing novel patterning approaches allowing both high-resolution and low-cost. Among other alternative methods, soft lithographies, such as microcontact printing, microtransfer molding, and microfluidics [1-3], are stimulating an increasing interest for their cheapness, good patterning performances, and unequalled flexibility in terms of the materials to be used.

The microfluidic lithography or micromolding in capillaries (MIMIC) was proposed as alternative route to microfabrication in 1995 by Whitesides and coworkers [1].

In its original formulation [2], it is based on the penetration of a prepolymer or of a suitably diluted polymer solution into a hydraulically connected networks of elastomeric channels, thus leading to the formation of a polymeric mask which can be used in subsequent lithography steps on the underlying substrate. In addition, MIMIC exhibits a very high flexibility towards the employable material, being applicable to bio-molecules, protein solutions, and drug deliveries [3, 4]. For these reasons, microfluidic lithography has raised increasing interest, both as an alternative, easy and cheap

\footnotetext{
* Corresponding author. Tel: +39-0832-298146; fax: +39-0832

298238; E-mail: dario.pisignano@unile.it
}

microfabrication technology, and for its application to fluidic-based devices. Indeed, the microfluidic technologies are the enabling factor for lab-on-chip devices allowing reduced consumption and waste of reagents and samples and increased operational speed [5, 6]. Many microelectronic and optoelectronic components have been realised by microfluidic lithographies, such as both inorganic [7] and organic [8] field effect transistors, optically amplified polymeric waveguides, [9] and directional carbon nanotube strings [10]. Recently developed fluidic devices include entropic traps for the separation of DNA molecules [11], complex chips integrating thousands of micromechanical valves and chambers [12], and microfluidic rectifiers [13].

However, an intrinsic problem of microfluidics is the slowness of the fluid penetration, especially when geometries with long capillary channels are used. Both for increasing the throughput of microfluidic-based lithographies, and for investigating the fluid dynamics within the micro-channels in depth, methods to directly observe the penetration dynamics are needed.

In this study, we monitored in real-time the penetration of a polyurethane fluid within microfluidic channels, showing how information about the capillarity process can be extracted by such direct observation,. In particular, the proposed integration of microfabricated side rulers within the silicon master structures is a straightforward way to exactly determine the time dependence of the covered length $(z, t)$ and of the filling rate $(v, t)$ under different 


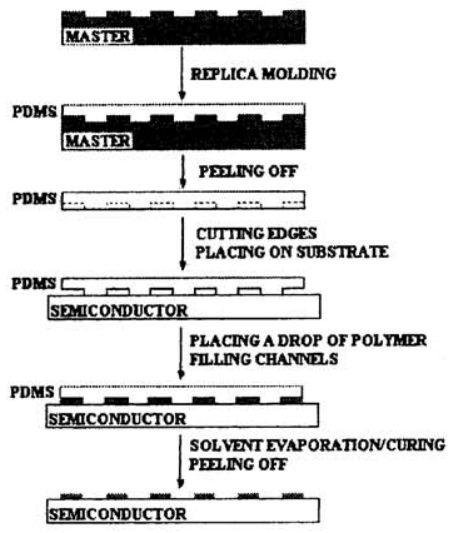

Fig. 1 Schematic diagram of the MIMIC procedure.

conditions, thus quantifying the fluid dynamics in capillarity-based chips, and leading to the possible optimisation of the liquid flow within microfluidic devices. Indeed, this approach allows one to quantitatively assess the strong dependence of the filling rate on the process environmental conditions (such as functionalisation, temperature, driving pressure, organic contaminants on the involved surfaces), and to discard in real-time the presence of impurities on employed substrates. We believe that these results will be of general interest for micro- and nanotechnology applications.

\section{Experimental}

Since soft lithography is based on the replication of an existing microstructure (i.e. the master) instead of on its fabrication [3], one first need to produce a template according to conventional patterning procedures. Our master structures were fabricated by photolithography on $\mathrm{Si} / \mathrm{SiO}_{2}$ (thickness $\cong 100 \mathrm{~nm}$ ) substrates. After the deposition of standard photo-sensitive resist (Clariant AZ5214E:AZ EBR Solvent 1:1), UV-exposure at $45 \mathrm{~W}$ and developing, we etched the thermal dioxide by using $\mathrm{NH}_{4} \mathrm{~F} / \mathrm{HF} / \mathrm{H}_{2} \mathrm{O}(6.6 \mathrm{~g} / 1.6 \mathrm{ml} / 10 \mathrm{ml})$. The so obtained $\mathrm{SiO}_{2}$ mask was then employed to etch Silicon by $5.9 \mathrm{M}$ solutions of $\mathrm{KOH}$ at $80^{\circ} \mathrm{C}$. Poly(dimethylsiloxane) (PDMS) was purchased from Dow Corning (Sylgard 184, A:B 1:9), cast on the masters and polymerised in situ at $140{ }^{\circ} \mathrm{C}$ for $15^{\text {, }}$ according to a standard replica molding process [3]. Elastomeric channels with section between 11 and $16 \mu \mathrm{m}$ were fabricated by this procesure.

Microfluidic lithography was carried out according to the scheme reported in Fig. 1. We cut the elastomeric mold by a razor blade to expose the micro-channels and placed it under its own weight on $\mathrm{Si}$ substrates, which were preliminary cleaned with an acetone and propanol cascade on a spinner. Finally, we deposited a drop of prepolymer (Polyurethane NOA72 purchased from Norland) at the edge of the mold in order to make the channels spontaneously filled by capillary action, cured the prepolymer by UVexposure and peeled off the PDMS replica. The motion of the fluid inside the channels was observed in real-time by optical microscopy. The sample temperature was varied by

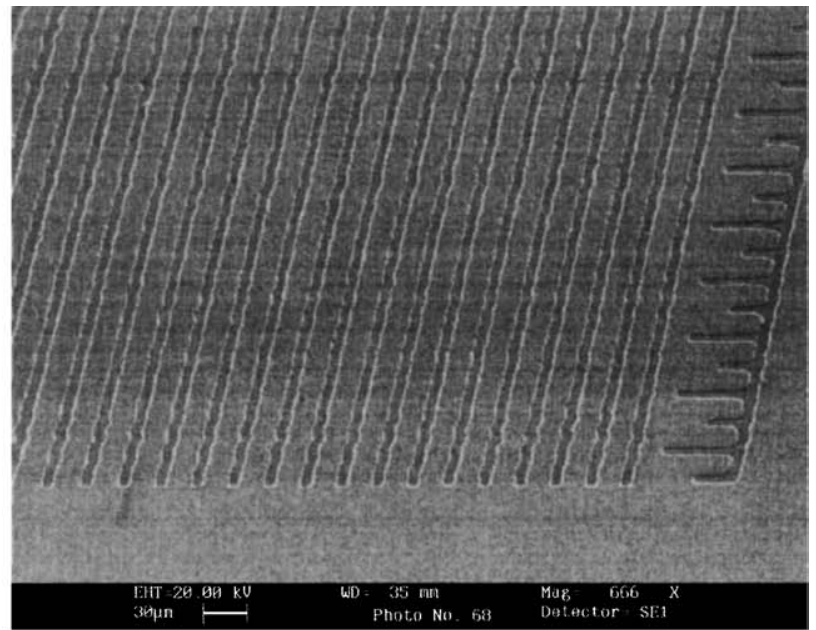

Fig. 2 SEM micrograph of the employed master. The period of the capillary grating is $30 \mu \mathrm{m}$.

a hot plate in contact with the bottom side of the substrate. Particular care was taken in order to avoid thermal gradients. Temperature was measured on the upper surface of the substrate (i.e. the surface in contact with the filling fluid), by a Pt100 sensor.

Finally, other capillarity conditions were obtained by functionalising the walls of our $\mu$-channels with $-\mathrm{OH}$ terminating groups, hydroxilating the $\mathrm{Si}$ surfaces by immersion in piranha solution and exposing the patterned side of PDMS to an $\mathrm{O}_{2}$ plasma treatment [14]. - $\mathrm{SH}$ functional terminations were obtained by the subsequent adsorption of 3-Mercaptopropyltrimethoxysilane from Ethanol solutions.

\section{Results and discussion}

The filling rate of the fluidic channels is proportional to the reciprocal length coordinate of the capillary, $z[2,15]$ :

$$
\frac{d z}{d t}=\frac{R \gamma \cos \Theta}{4 \eta z}
$$

where $\eta, R, \gamma$ and $\Theta$ indicate the liquid viscosity, the radius of the capillary, the liquid-vapour interfacial free energy of the fluid, and the contact angle between the liquid and the surface of the capillary, respectively. $\Theta$, whose cosine is proportional to the difference between the solid-liquid and the solid-vapour interfacial energies, determines whether the filling process is thermodynamically favoured, i.e. if the channels can be wetted by the polymer $(\cos \Theta>0)$ or not $(\cos \Theta<0)$. The time, $t$, needed for the filling process is proportional to the square length:

$$
t=\frac{\eta z^{2}}{2 \Delta p G}
$$

In Eq. (2), $\Delta p$ is the difference between the pressure which drives the capillary motion, while $G$ depends on the geometry of the channel. An intrinsic problem of this technique is therefore the slowness of the pattern formation. However, Eq. (1) and (2) imply that the operation temperature (i.e. $\eta$ ) and the functionalisation of the 


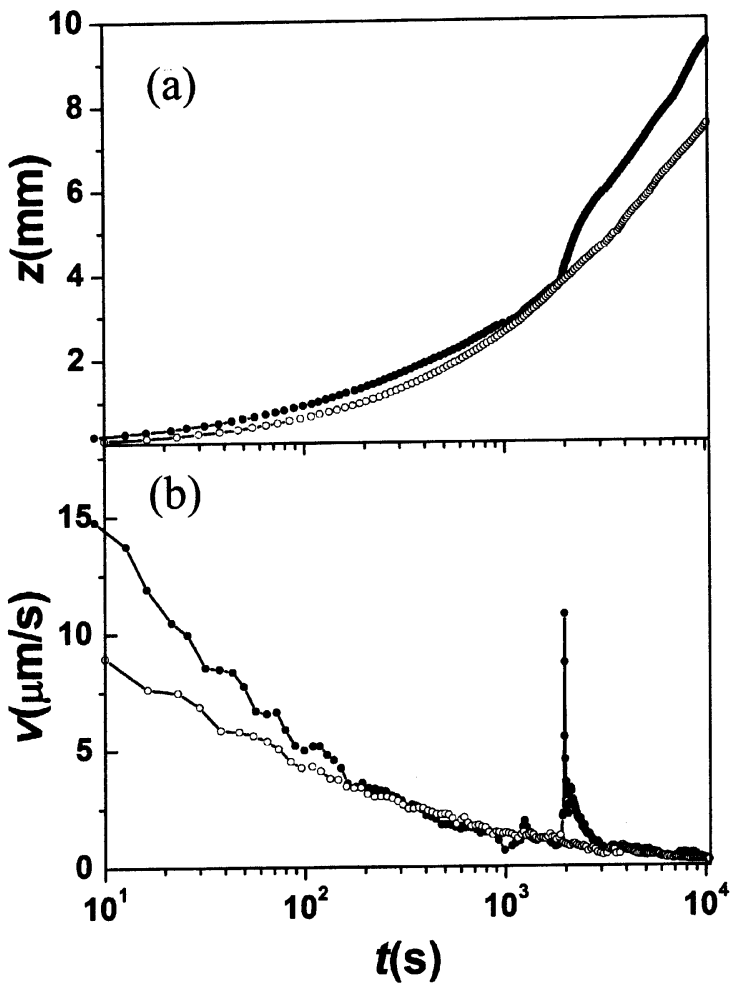

Fig. 3 Covered length, $z$ (a), and filling speed, $v(\mathrm{~b})$, of the capillary front vs. time, $t$, for a well-cleaned substrate (open circles) and a substrate presenting impurities around $z=4 \mathrm{~mm}$, i.e. $t=2 \times 10^{3} \mathrm{~s}$ (full circles). Note the corresponding anomalous increase of $z$ and $v$. The sample temperature is $19^{\circ} \mathrm{C}$.

capillary walls (i.e. on $\Theta$, namely on the effective $\Delta p$ ) directly affect the capillarity process, hence they can be varied aiming at better controlling the fluidic flows and possibly enhancing the penetration rate. We investigated both these approaches.

In our investigation we employed, as molds, gratings of about $19 \mu \mathrm{m}$ wide parallel stripes with a period of $30 \mu \mathrm{m}$ (Fig. 2). Moreover, in order to accurately measure the filling speed during the capillary process by optical microscopy, we integrated in the masters, side by side with the channels, a ruler with $45 \mu \mathrm{m}$ long major ticks separated by $100 \mu \mathrm{m}$, and minor ticks ( $17 \mu \mathrm{m}$ long) separated by 50 $\mu \mathrm{m}$. Since PDMS is transparent to light in the wavelength range between 300 and $800 \mathrm{~nm}$, this allowed us to exactly determine the length, $z$, covered by the polymer fluid as a function of the filling time, $t$ [16]. This is a flexible and straightforward approach, which can be easily extended to any kind of planar pattern. A home-made software was employed, allowing to collect hundreds of $(z, t)$ points, hence allowing one to carefully follow the capillary dynamics also at the beginning of the penetration process, when filling speeds of the order of tens of $\mu \mathrm{m} / \mathrm{s}$ have been usually registered.

A typical room-temperature capillary curve $(z, t)$ is shown in Fig. 3a (open dots). It takes a time $t_{l}=16500 \mathrm{~s}$ for NOA72 to completely fill a $9 \mathrm{~mm}$ long channel at $19^{\circ} \mathrm{C}$. Increasing temperature up to $30^{\circ} \mathrm{C}$, we could appreciate a strong decrease of the filling time down to $t_{2}=2765 \mathrm{~s}$. By differentiating the $(z, t)$ experimental curve, we could obtain the time-dependence of the penetration rate $(v, t)$. Upon Polyurethane flowing inside the capillaries at room temperature, $v$ decreases from 10.0 to less than $0.2 \mu \mathrm{m} / \mathrm{s}$. At $30^{\circ} \mathrm{C}$, a similar decrease was observed from 18.6 to 1.6 $\mu \mathrm{m} / \mathrm{s}$. Therefore, by increasing the operation temperature of about $10^{\circ} \mathrm{C}$, a residual penetration speed 8 times larger than that at room temperature was found at the end of our microchannels. A maximum increase of the overall microfluidic throughput by a factor around 60 was observed in our study, opening the door to the realisation of large-area devices by microfluidic lithography [15].

Moreover, the possible presence of organic impurities either on the elastomeric element, or on the Si substrates was found to heavily affect the microfluidic motion. This finding agrees with the dependence of the penetration rate on the mutual interfacial energies (Eq. 1). Under these conditions, the fluidic penetration is no longer described by a smooth behaviour, such that of Eq. 2. Indeed, we found that the organic contaminants due to non-perfectly cleaned surfaces generally determine abrupt discontinuities of the filling rate (see the acceleration from 0.9 to $10.8 \mu \mathrm{m} / \mathrm{s}$ at about 2000 s in Fig. 3b), whereas the capillary penetrations on carefully cleaned Silicon substrates exhibit well behaved $(z, t)$ and $(v, t)$ curves. Therefore, the real-time monitoring allows to determine the exact location of organic impurities and their extension on the substrate, and to rapidly discard dirty wafers, thus contributing to increase the lithography throughput.

With increasing the temperature up to $80^{\circ} \mathrm{C}$ the time to fill $9 \mathrm{~mm}$-long channels reduces by over a factor of 60 . Moreover, one observes that a rapid increase of the filling speed occurs mainly just above room temperature, as the lithography throughput increases by one order of magnitude between 19 to $30^{\circ} \mathrm{C}$. This behaviour can be explained considering the temperature dependence of the filling speed implicit in Eq. (1), which is mainly due to the viscosity, $\eta$. Indeed, as the temperature increases, the mobility of the molecules constituting the liquid dramatically increases, thus inducing an abrupt decrease of both the structural characteristic relaxation time and the viscosity. The temperature dependence of $\eta$ for glass-forming liquids, is usually well-described by a Vogel-Fulcher-Tamman law (VFT) [17]:

$$
\eta(T)=\eta_{0} \exp \left(\frac{D T_{0}}{T-T_{0}}\right),
$$

where $D$ is referred to as strength parameter and $T_{0}$ is the temperature for which Eq. (3) shows a divergence in the polymer structural relaxation time and, consequently, in the viscosity. The strength of the glass-forming fluid is directly related to the stability of the system at temperatures above the glass transition [18]. Fragile systems (low values of $D$ ) are characterised by larger variations of their thermodynamic properties upon varying temperatures. For this reason, they are the most suitable compounds for highthroughput soft lithography, since a large decrease of the viscosity, hence a high wettability and conformability to the elastomeric features, can be achieved by slight increase of temperature. 


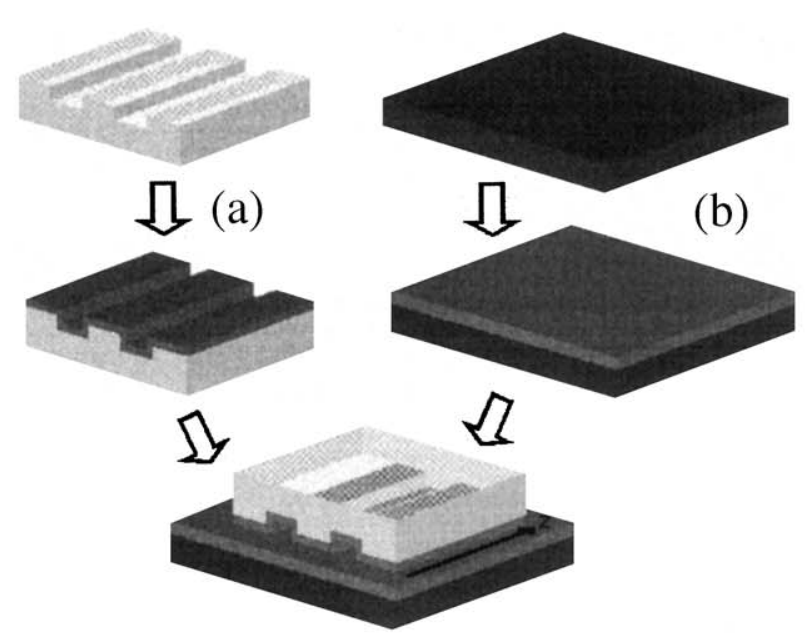

Fig. 4 Scheme of the microfluidic process by channels functionalised for high throughput (features not in scale). (a) and (b): Functionalisation treatment of the replica and the substrate, respectively. The filling length coordinate, $z$, is indicated on the assembled sample.

Finally, since the filling polyurethane liquid is also hydrophilic [19], the lithography performances can also be improved by customizing the capillary surfaces (i.e. the contact angle, $\Theta$ ) by proper polar moieties. This can be achieved by reactive plasma or incubation treatments: In our studies, we covered the walls of our micro-channels with $-\mathrm{OH}$ and $-\mathrm{SH}$ functionalities, obtaining an improvement of the microfluidic throughput by over a factor of 3 [20]. For instance, it takes a time $t_{O H}=2845$ and $t_{S H}=2040 \mathrm{~s}$ to fill a $1 \mathrm{~cm}$ long channel covered by $-\mathrm{OH}$ and -SH groups, respectively (the corresponding penetration time for the same untreated micro-capillaries was about $6380 \mathrm{~s}$ ). The measured filling rates at $z=1 \mathrm{~mm}$ and $z=10$ $\mathrm{mm}$ were about 9.0 and $0.7 \mu \mathrm{m} / \mathrm{s}, 12.2$ and $2.0 \mu \mathrm{m} / \mathrm{s}$, and 29.1 and $2.7 \mu \mathrm{m} / \mathrm{s}$ for untreated, $-\mathrm{OH}$ terminated and $-\mathrm{SH}$ terminated channels, respectively. The penetration speed was so increased by a factor between 2.8 and 41.6, depending on the chosen hydrophilic groups and the different sample region. In addition, no region was usually observed, exhibiting the singular behaviour shown in Fig. $3 c$, which evidences the uniformity of the achieved layers on the walls of the microchannels.

As shown in Fig. 4, this approach is complementary to the inking of the elastomeric elements usually carried out in microcontact printing, since it exploits the functionality provided by the recessed regions of the stamp (forming the walls of the micro-channels), instead of using the delivered molecules by the protrusion of the replica.

\section{Conclusion}

The time dependence of the covered length $(z, t)$ and of the filling rate $(v, t)$ under different conditions of temperature and interfacial energies was determined with optical microscopy, by real-time observation of the microfluidic motion. We believe that the quantitative determination of the dependence of the filling rate on the different process environmental variables, and the real-time assessment of the presence of impurities on the employed substrates can both provide a deeper knowledge of the fluid dynamics in micro-capillaries, and open the way to novel and more efficient microfluidic architectures.

\section{References}

[1] E. Kim, Y. Xia G. M. Whitesides, Nature 376 (1995) 581.

[2] E. Kim, Y. Xia, G. M. Whitesides, J. Am. Chem. Soc. 118 (1996) 5722

[3] Y. Xia, G. M. Whitesides, Angew. Chem. Int. Ed. 37 (1998) 550.

[4] E. Delamarche, A. Bernard, H. Schmid, A. Bietsch, B. Michel, H. Biebuyck, J. Am. Chem. Soc. 120 (1998) 500

[5] T. Vilkner, D. Janasek, A. Manz, Anal. Chem. 76 (2004) 3373.

[6] M. Heckele, W. K. Schomburg, J. Micromech. Microeng. 14 (2004) R1.

[7] N. L. Jeon, J. Hu, G. M. Whitesides, M. K. Erhardt, R. Nuzzo, Adv. Mater. 17 (1998) 1466.

[8] W. S. Beh, I. T. Kim, D. Qin, Y. Xia, G. M. Whitesides, Adv. Mater. 11 (1999) 1038.

[9] X.-M. Zhao, A. Stoddart, S. P. Smith, E. Kim, Y. Xia, M. Prentiss, G. M. Whitesides, Adv. Mater. 8 (1996) 420. P. Yang, G. Wirnsberger, H. C. Huang, S. R. Corsero, M. D. McGehee, B. Scott, T. Deng, G. M. Whitesides, B. F. Chmelka, S. K. Buratto, G. D. Stucky, Science 287 (2000) 465

[10] J. Chen, W. A. Weimer, J. Am. Chem. Soc. 124 (2002) 758.

[11] J. Han, H. G. Craighead, Science 288 (2000) 1026.

[12] T. Thorsen, S. J. Maerkl, S. R. Quake, Science 298 (2002) 580.

[13] A. Groisman, S. R. Quake, Phys. Rev. Lett. 92 (2004) 94501.

[14] G. S. Ferguson, M. K. Chaudhury, H. A. Biebuyck, G. M. Whitesides, Macromolecules 26 (1993) 587. G. S. Ferguson, M. K. Chaudhury, G. B. Sigal, G. M. Whitesides, Science 253 (1991) 776. M. K. Chaudhury, G. M. Whitesides, Science 256 (1992) 1230.

[15] D. Myers, Surfaces, Interfaces and Colloids; Wiley-VCH Publishers, New York 1999.

[16] D. Pisignano, E. Sariconi, M. Mazzeo, G. Gigli, R. Cingolani, Adv. Mater. 21 (2002) 1565.

[17] H. Vogel, Phys. Z 22 (1921) 645. G. S. Fulcher, J. Am. Ceram. Soc.8 (1925) 339. G. Tamman, W. Hesse Z Anorg. Allg. Chem. 156 (1926) 245.

[18] R. Bohmer, C. A. Angell, Phys. Rev. B 45 (1992) 10091.

[19] Y. Xia, J. Tien, D. Qin, G. M. Whitesides, Langmuir 12 (1996) 4033.

[20] D. Pisignano, F. Di Benedetto, L. Persano, G. Gigli, R. Cingolani, Langmuir 20 (2004) 4802. 